\title{
Qualitative Grading as a Tool in the Management of Multilevel Lumbar Spine Stenosis
}

\author{
Shardul Madhav Soman ${ }^{1}$, Jimmy Chokshi ${ }^{1}$, Naitik Chhatrala ${ }^{2}$, \\ Gulam Haider Tharadara ${ }^{2}$, Mukund Prabhakar ${ }^{2,3}$ \\ ${ }^{1}$ Department of Orthopaedics, Government Spine Institute and Paraplegia Hospital, Ahmedabad, India \\ ${ }^{2}$ Department of Orthopaedics, BJ Medical College and Civil Hospital, Ahmedabad, India \\ ${ }^{3}$ Department of Orthopaedics, Government Spine Institute and Paraplegia Hospital, Ahmedabad, India
}

Study Design: This is a prospective study that was undertaken at a single centre and involved 80 consecutive patients diagnosed with lumbar spinal stenosis (LSS).

Purpose: The aim of the study was to assess the efficacy of a qualitative grading system as seen on magnetic resonance imaging (MRI) as a tool in the management of multilevel LSS.

Overview of Literature: LSS diagnosis is clinical but is usually radiologically supplemented. However, there are often multilevel radiological findings with non-specific or atypical clinical features. We used a qualitative grading system to help in the decision-making process of the management of patients with multilevel LSS.

Methods: 80 patients with LSS were treated with decompression and prospectively followed-up for a minimum of 12 months. All had failed conservative treatment. Qualitative grading of LSS severity was based on the dural sac in T2 weighted axial MRI images at all disc levels and was done from L1-2 to $L 5-S 1$ ( $n=400)$. Functional outcome was assessed using the Oswestry disability index (ODI). Results: The mean patient age was 56.6 years, with a gender ratio of 0.6:1. Forty patients had degenerative LSS and 40 had degenerative spondylolysthesis. A total of 178 levels were decompressed, the majority of which were L4-L5 (43.82\%), followed by L5-S1 $(41.57 \%)$. According to our qualitative grading system, grade D stenosis $(53.93 \%)$ was decompressed most frequently, followed by grade C stenosis $(41.57 \%)$. The average preoperative ODI score was $58.55 \%$, which later reduced to $19.15 \%$. Seventy percent of patients achieved excellent results, whereas $30 \%$ achieved good results.

Conclusions: Morphological grading is a useful tool in decision making in surgery for multilevel LSS. Grade C and D stenosis should be decompressed, whereas A and B should not be, unless clinically justified

Keywords: Decompression; Lumbar spinal stenosis; Multilevel; Grading

\section{Introduction}

Verbiest [1-10] was the first person to coin the term spinal stenosis and attributed its cause to the narrowing of the spinal canal. Later, Kirkaldy-Willis et al. [11-13] explained the degenerative cascade in the lumbar spine, which led to the canal narrowing. Lumbar spinal stenosis (LSS) can be a disabling condition. After the failure of conservative treatment, surgery is the next mode of management. In patients aged more than 65 years, LSS is the most common

Received Jul 19, 2016; Revised Aug 18, 2016; Accepted Sep 19, 2016

Corresponding author: Shardul Madhav Soman

Department of Orthopaedics, BJ Medical College and Civil Hospital, Ahmedabad,

4 th Floor Government Spine Institute, Ahmedabad, Gujarat India 361008

Tel: +918980844549, E-mail: somanshardul@gmail.com 
underlying cause of lumbar surgery [14,15]. LSS diagnosis is made clinically and supplemented radiologically. However, there are often multilevel radiological findings with non-specific or atypical clinical features. Many radiological criteria have been proposed to facilitate the diagnosis, but none are ideal. Here we used a qualitative grading system to help in the decision-making process of the management of patients with multilevel LSS.

\section{Materials and Methods}

Eighty consecutive patients with LSS were treated with decompression and prospectively followed-up for a minimum of 1 year. All patients had unilateral or bilateral leg pain, neurological claudication with or without neurological deficit, and back pain. All had failed conservative treatment, which comprised 6 weeks of structured physical therapy, nonsteroidal anti-inflammatory drugs for pain, and epidural steroids, as needed. Patients with previous spine surgery, referred leg pain due to other pathologies such as polyneuritis, or severe degenerative scoliosis with Cobb's angle of $>30^{\circ}$ were excluded. All had preoperative standing anteroposterior (AP), lateral, and dynamic radiographs, along with $\mathrm{T} 1$ and $\mathrm{T} 2$ weighted axial and sagittal magnetic resonance imaging (MRI). Sagittal T1-weighted spin-echo, sagittal T2-weighted fast spinecho, and axial T2-weighted fast spin-echo lumbar spine images were acquired using magnets operating at the field strength of 2.0 T. Qualitative grading of LSS severity was based on the dural sac in T2 weighted axial MRI images at all disc levels (Fig. 1) and was done by a senior spine surgeon who was not involved in the study. The grading was based on the cerebro-spinal fluid (CSF)/rootlet ratio as observed in axial T2 images and was conceived following observation of different patterns according to which the rootlets were disposed within the dural sac while the patient rested supine during MRI acquisition. This was done at all disc levels from L1-L2 to L5-S1 $(n=400)$. Functional outcome was assessed using the Oswestry disability index (ODI) (Fig. 2).

\section{Results}

The mean patient age was 56.6 years, and the male to female ratio was 0.6:1. All patients were prospectively followed-up for a minimum of 1 year. Forty patients had degenerative LSS and the other forty had degenerative spondylolysthesis. A total of 178 levels were decompressed, of which the majority were L4-L5 (43.82\%), followed by L5-S1 (41.57\%) (Table 1). According to qualitative grading, grade D stenosis (53.93\%) was decompressed most frequently, followed by grade C $(41.57 \%)$ and grade B (4.49\%) stenosis (Table 2). The most common qualitative grade at L1-L2 was A3, at L2-L3 and L3-L4 was

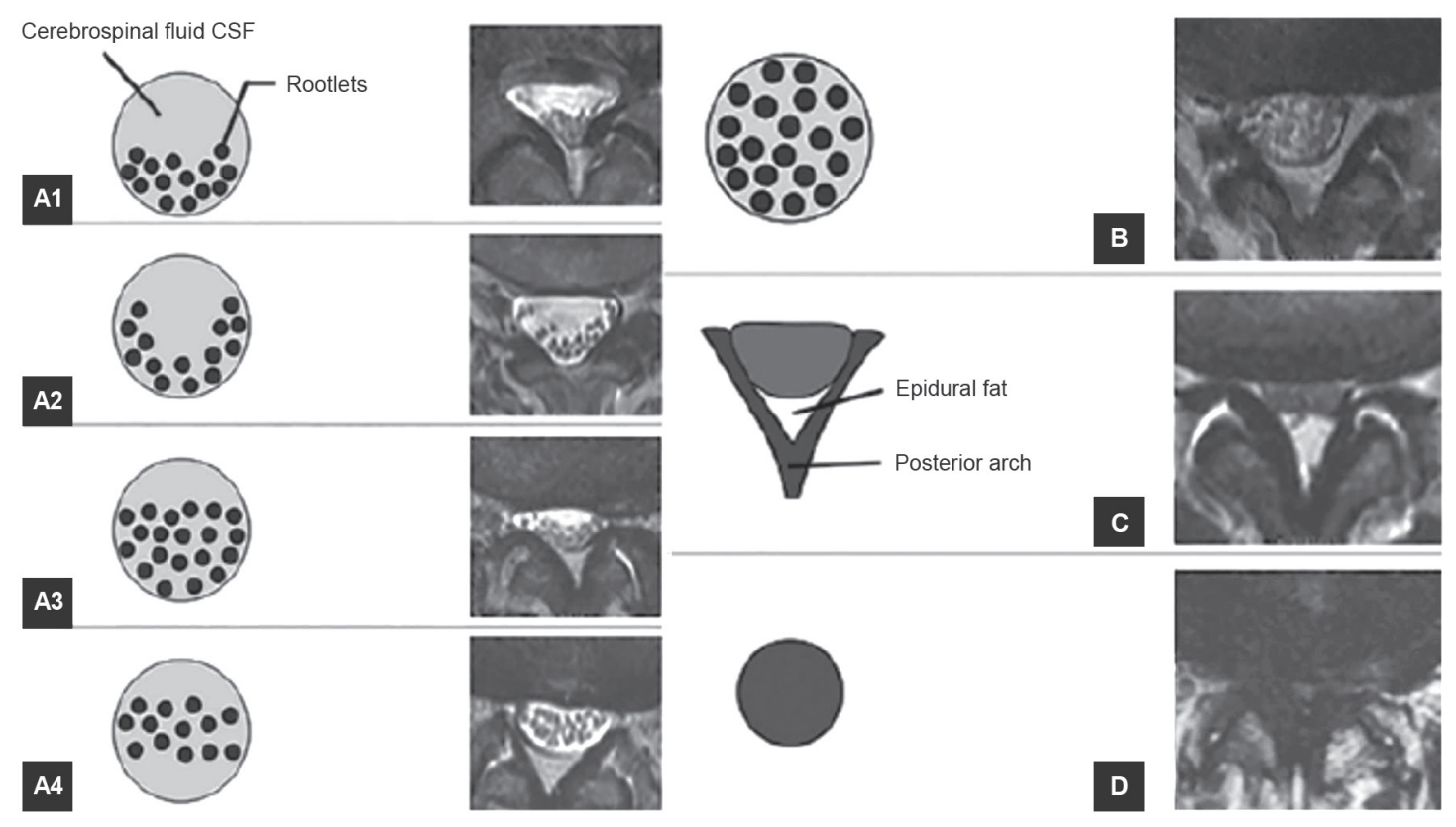

Fig. 1. Morphological grading based on magnetic resonance imaging axial T2 images. CSF, cerebro-spinal fluid. 

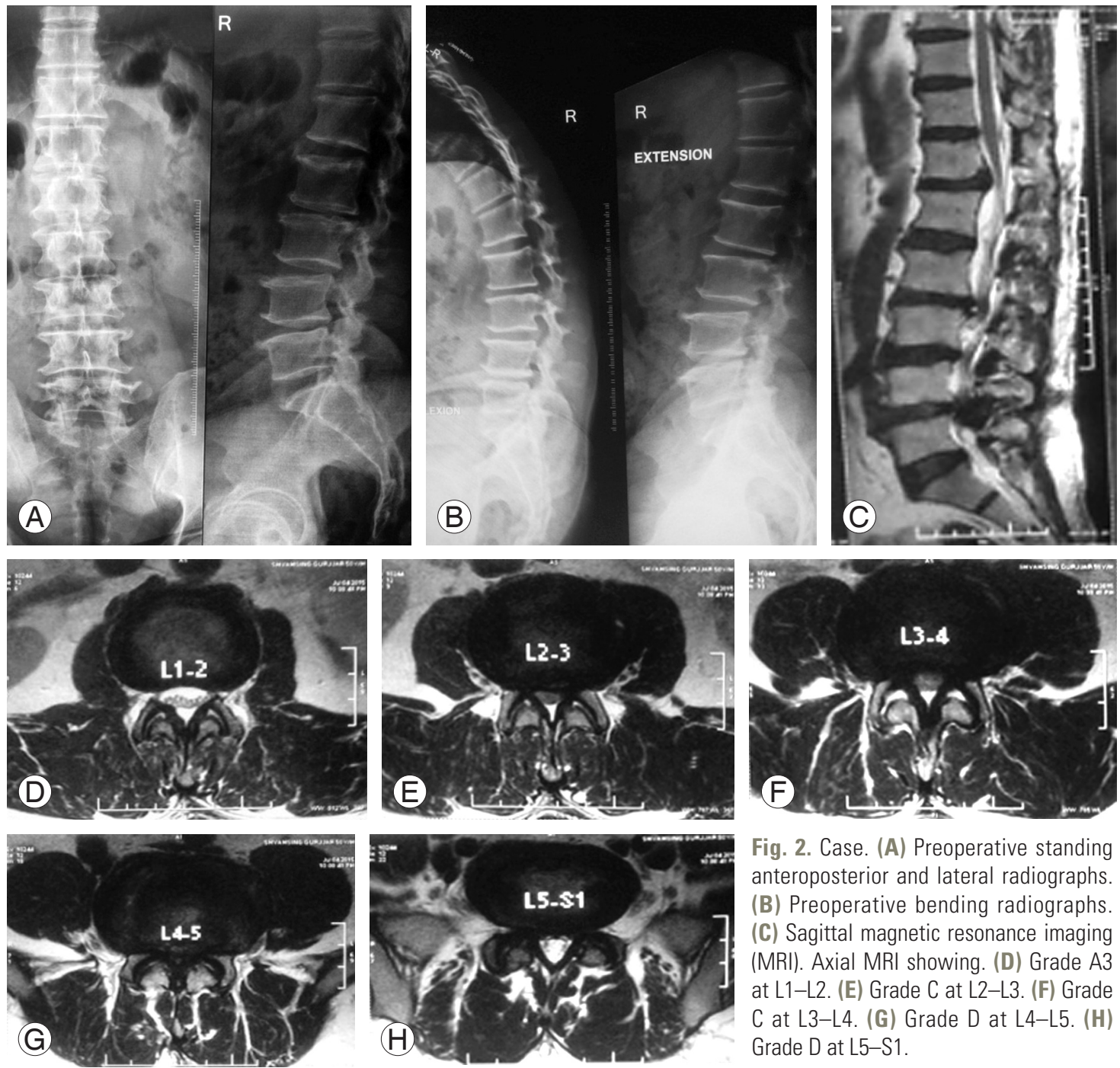

Fig. 2. Case. (A) Preoperative standing anteroposterior and lateral radiographs. (B) Preoperative bending radiographs. (C) Sagittal magnetic resonance imaging (MRI). Axial MRI showing. (D) Grade A3 at L1-L2. (E) Grade C at L2-L3. (F) Grade C at L3-L4. (G) Grade D at L4-L5. (H) Grade D at L5-S1.

Table 1. Levels decompressed

\begin{tabular}{lc} 
Level & No. of levels decompressed \\
L1-L2 & 0 \\
L2-L3 & $2(1.12)$ \\
\hline L3-L4 & $24(13.48)$ \\
\hline L4-L5 & $78(43.82)$ \\
\hline L5-S1 & $74(41.57)$ \\
\hline Total & $178(100)$ \\
\hline
\end{tabular}

Values are presented as number (\%).

$\mathrm{B}$, at L4-L5 were C and D, and at L5-S1 was D (Table 3). Surgical procedures performed included interbody fusion, laminectomy, and laminectomy with posterolateral instrumented fusion with local posterior iliac crest bone graft (Table 4). The average duration of hospitalisa-
Table 2. Levels decompressed as per qualitative grading

\begin{tabular}{lc} 
Qualitative grade & $\begin{array}{c}\text { No. of qualitative level } \\
\text { decompressed }\end{array}$ \\
\hline A1 & 0 \\
\hline A2 & 0 \\
A3 & 0 \\
\hline A4 & 0 \\
\hline B & $8(4.49)$ \\
\hline C & $74(41.57)$ \\
\hline D & $96(53.93)$ \\
\hline Total & $178(100)$ \\
\hline
\end{tabular}

Values are presented as number (\%).

tion was 6.55 days, with the majority of patients requiring 4-6 days of postoperative hospitalisation (65\%). Average 
Table 3. Qualitative grade at each level

\begin{tabular}{lccccccc} 
Level in lumbar spine & A1 & A2 & A3 & A4 & B & C & D \\
\hline L1-L2 & 4 & 16 & 30 & 22 & 8 & 0 & 0 \\
$\mathrm{~L} 2-\mathrm{L} 3$ & 4 & 12 & 8 & 16 & 34 & 6 & 0 \\
$\mathrm{~L} 3-\mathrm{L} 4$ & 0 & 0 & 4 & 0 & 56 & 18 & 2 \\
$\mathrm{~L} 4-\mathrm{L} 5$ & 0 & 0 & 0 & 0 & 2 & 40 & 38 \\
$\mathrm{~L} 5-\mathrm{S} 1$ & 0 & 0 & 0 & 0 & 6 & 18 & 56 \\
\hline
\end{tabular}

Table 4. Surgical procedure undertaken

\begin{tabular}{lcc} 
Surgical procedure & No. of patients & No. of levels \\
Interbody fusion & 48 & 96 \\
Laminectomy & 28 & 70 \\
Laminectomy with instrumented posterolateral fusion & 4 & 12 \\
\hline Total & 80 & 178 \\
\hline
\end{tabular}

Table 5. Comparison of ODI scores with other studies

\begin{tabular}{lcc} 
Study & Mean preoperative ODI score (\%) & Mean postoperative ODI score (\%) \\
\hline Park et al. [16] & 55 & 16 \\
\hline Deutsch et al. [17] & 57 & 18 \\
Ganz [18] & 56 & 20 \\
Our study & 58.5 & 19.15 \\
\hline
\end{tabular}

ODI, Oswestry disability index.

preoperative ODI score was $58.55 \%$, which later reduced to $19.15 \%$. Seventy percent patients had excellent results and $30 \%$ had good results. The patients were graded in terms of their improvement in ODI score: 0\%-20\% (excellent, minimal disability), 21\%-40\% (good, moderate disability), 41\%-60\% (fair, severe disability), and $>60 \%$ (poor, crippled disability). Of the patients with excellent results, $85.71 \%$ had leg pain for $<1$ year, while all patients having good results had leg pain for $>1$ year. Our study was at par with other studies in terms of ODI score $(p<0.05)$ (Table 5). Four patients developed complications, two had an infection, and the other two developed implant loosening.

\section{Discussion}

LSS is a fairly common condition, with an incidence of $1: 1,000$; this ratio has increased due to an increase in life expectancy [19-21]. With the introduction of new diagnostic methods in clinical practice, new radiological criteria for spinal canal assessment have been proposed, along with areas and levels of measurement. MRI is considered the "gold standard" for LSS diagnosis considering its ability to visualize Roentgen-negative soft tissues.

The present classification system, which has the advantage of being easily applicable in daily clinical practice and does not involve the use of specific measuring tools, takes into account the CSF content of the canal. It appears to better reflect the definition of stenosis than numerical values of surface measurements alone, as this system considers the degree of impingement of the dural sac contents. It has been reported to have a moderate to substantial agreement amongst observers [22].

Barz et al. [23] introduced the use of the nerve root sedimentation sign and Schizas et al. [22] introduced qualitative morphological grading as a means to aid clinicians in diagnosing LSS.

As per our study, morphological grading of $\mathrm{C}$ and $\mathrm{D}$ stenosis was more common at lower lumbar levels because acquired stenosis due to degenerative changes is more common there. The most common decompressed level 
was L4-L5 (43.82\%), followed by L5-S1 (41.57\%), which is similar to the results reported by Nath et al. [24] and the SPORTS trial [25] $(p<0.05)$. L4-L5 and L5-S1 involvement is due to acquired stenosis most commonly at the first and second levels of the anatomical segment, because of early degenerative changes such as hypertrophy of ligamentum flavum, facet joint arthropathy, disc osteophyte complex, and spondylolisthesis. The spinal canal at L4 and L5 is of a trefoil shape, and the L5 foramen is the smallest of all lumbar vertebral foramen; thus, it is more prone to stenosis with degenerative changes.

There was a strong association between the levels that had surgical intervention and their preoperative qualitative grading on MRI. There were 178 operated levels in total ( $\mathrm{n}=400$ ), of which 8 had grade $B, 74$ had grade $C$, and 96 had grade D stenosis. Hence, in the majority of cases, grades $\mathrm{C}$ and $\mathrm{D}$ were decompressed, and we obtained 56 excellent and 24 good results, according to the ODI score postoperatively assessed at 12 months. No poor results were obtained. These results reiterate that the presence of grade $\mathrm{C}$ and $\mathrm{D}$ stenosis should be assessed before surgeons make a final decision on surgical intervention. The absence of grade C and D stenosis at a surgical level should make a surgeon seriously review their decision making or at least perform further diagnostic tests that support their decision.

Grade A and B stenosis, however, do not necessarily imply that the level need not be decompressed. In our study, 8 levels with grade B stenosis were decompressed. Thus, grade A and B stenosis do not necessarily eliminate the option of surgical treatment but instead require a surgeon to perform further diagnostic testing to decide for or against surgery.

Considering the fact that the majority of patients achieve better clinical outcomes after grade $\mathrm{C}$ and $\mathrm{D}$ decompression, grade $\mathrm{C}$ and $\mathrm{D}$ stenosis could be accountable for clinical signs and symptoms. This establishes an indirect correlation between the clinical picture and morphological grading.

Usually, LSS simultaneously affects multiple contiguous levels. However, not every level needs to be decompressed and surgical decision making should take into account the patients' clinical pictures, pattern of referred pain on physical examination, and other diagnostic measures. It can therefore be concluded that grade $\mathrm{C}$ and $\mathrm{D}$ decompression could lead to better results and we can predict the prognosis, making our grading system an important tool in surgical decision making.

Schizas et al. [22], Amundsen et al. [26], Sirvanci et al. [27], and Sigmundsson et al. [28] reported no or poor correlation among the degree of canal stenosis, dural cross sectional area, morphology grade on MRI with clinical symptoms, ODI score, functional outcomes measured in form of Short Form-36, European Quality of Life scale, walking distance, and clinical outcomes. None of these studies assessed morphologic characteristics and they were limited in trying to establish a relationship among measured parameters and symptoms or functional status. Our study on the other hand establishes an indirect correlation between the clinical picture and morphological grading.

In our study, $65 \%(n=52)$ patients required $4-6$ days of postoperative hospital stay, with a mean postoperative hospital stay of 6.5 days; these results are similar to those of Zheng et al. [29] ( $p<0.05)$. Increasing age, multilevel decompression/fusion, infection, operative correction of degenerative scoliosis, excessive body weight, and other comorbid conditions are important predictors of a longer hospital stay [29].

In our study, 56 patients had excellent results. From this group, 48 (85.71\%) had preoperative leg pain for less than a year. Moreover, 12 had good results, all of which had preoperative leg pain for more than a year $(p<0.05)$, which is similar to the results reported by Sigmundsson et al. [28]. A longer duration of leg pain is an inferior outcome due to permanent pathophysiological changes occurring to spinal roots. Our results are similar to those of Ganz [18], Park and Foley [16], and Deutsch and Musacchi [17]. Excellent outcomes were achieved due to good surgical decision making by means of the sedimentation sign and qualitative morphological grading, along with sincere preoperative history taking and physical examination.

One of the limitations of this study was that different patients underwent different surgical procedures, including decompression with or without fusion procedure. The patients who underwent fusion procedures had instability on dynamic films or severe facetal degeneration, making the specified surgery a requirement. We are however unsure whether this influenced the outcome as no such correlation was observed among different procedures. Koc et al. [30] reported a $10.3 \%$ overall revision rate in long-term follow-up of LSS patients. The rate of reoperation is determined by numerous factors. It is our belief that the reoperation rate could be decreased by better initial diagnosis, 
thereby more accurately identifying which levels do and do not require surgical intervention using morphological grading.

\section{Conclusions}

As per our results, morphological grading is a useful tool in aiding surgical decision making for multilevel LSS. This tool will go a long way in rationalizing the treatment. Grade C and D stenosis generally fail conservative treatment, and these should be decompressed, while grade $\mathrm{A}$ and $\mathrm{B}$ stenosis respond well to conservative management; hence, this should be the primary treatment modality for this stage. Clinical findings should, however, also be considered, as seen with grade B stenosis that also required decompression. In future, this study should be conducted at a larger scale and with longer follow-up duration.

\section{Conflict of Interest}

No potential conflict of interest relevant to this article was reported.

\section{References}

1. Verbiest $\mathrm{H}$. A radicular syndrome from developmental narrowing of the lumbar vertebral canal. J Bone Joint Surg Br 1954;36:230-7.

2. Verbiest H. Further experiences on pathologic influence of a developmental stenosis of the lumbar vertebral canal. J Bone Joint Surg Br 1956;38:576-83.

3. Verbiest H. Spondylolisthesis: the value of radicular signs and symptoms. J Intern Coll Surg 1963;39:46181.

4. Verbiest H. Pathomorphic aspects of developmental lumbar stenosis. Orthop Clin North Am 1966;6:17796.

5. Verbiest H. Pathomorphologic aspects of developmental lumbar stenosis. Orthop Clin North Am 1975; 6:177-96.

6. Verbiest H. Unilateral lumbo-sacral radicular symptoms due to sequestrated disc material in the spinal canal. Cesk Neurol 1968;31:93-101.

7. Verbiest H. Chapter 16. Neurogenic intermittent claudication in cases with absolute and relative stenosis of the lumbar vertebral canal (ASLC and RSLC), in cases with narrow lumbar intervertebral foram- ina, and in cases with both entities. Clin Neurosurg 1973;20:204-14.

8. Verbiest $H$. The treatment of lumbar spondyloptosis or impending lumbar spondyloptosis accompanied by neurologic deficit and/or neurogenic intermittent claudication. Spine (Phila Pa 1976) 1979;4:68-77.

9. Verbiest H. Stenosis of the lumbar vertebral canal and sciatica. Neurosurg Rev 1980;3:75-89.

10. Verbiest H. Developmental stenosis of the bony lumbar vertebral canal. Acta Orthop Belg 1987;53:37387.

11. Kirkaldy-Willis WH, Paine KW, Cauchoix J, McIvor G. Lumbar spinal stenosis. Clin Orthop Relat Res 1974;(99):30-50.

12. Kirkaldy-Willis WH, Wedge JH, Yong-Hing K, Reilly J. Pathology and pathogenesis of lumbar spondylosis and stenosis. Spine (Phila Pa 1976) 1978;3:319-28.

13. Kirkaldy-Willis WH, Wedge JH, Yong-Hing K, Tchang S, de Korompay V, Shannon R. Lumbar spinal nerve lateral entrapment. Clin Orthop Relat Res 1982;(169):171-8.

14. Deyo RA, Ciol MA, Cherkin DC, Loeser JD, Bigos SJ. Lumbar spinal fusion. A cohort study of complications, reoperations, and resource use in the Medicare population. Spine (Phila Pa 1976) 1993;18:1463-70.

15. Deyo RA, Gray DT, Kreuter W, Mirza S, Martin BI. United States trends in lumbar fusion surgery for degenerative conditions. Spine (Phila Pa 1976) 2005; 30:1441-5.

16. Park P, Foley KT. Minimally invasive transforaminal lumbar interbody fusion with reduction of spondylolisthesis: technique and outcomes after a minimum of 2 years' follow-up. Neurosurg Focus 2008;25:E16.

17. Deutsch H, Musacchio MJ Jr. Minimally invasive transforaminal lumbar interbody fusion with unilateral pedicle screw fixation. Neurosurg Focus 2006;20: E10.

18. Ganz JC. Lumbar spinal stenosis: postoperative results in terms of preoperative posture-related pain. J Neurosurg 1990;72:71-4.

19. Grenier N, Kressel HY, Schiebler ML, Grossman RI, Dalinka MK. Normal and degenerative posterior spinal structures: MR imaging. Radiology 1987;165:51725.

20. Lee CK, Rauschning W, Glenn W. Lateral lumbar spinal canal stenosis: classification, pathologic anatomy and surgical decompression. Spine 1988;13:313-20. 
21. Strojnik T. Measurement of the lateral recess angle as a possible alternative for evaluation of the lateral recess stenosis on a CT scan. Wien Klin Wochenschr 2001;113 Suppl 3:53-8.

22. Schizas C, Theumann N, Burn A, et al. Qualitative grading of severity of lumbar spinal stenosis based on the morphology of the dural sac on magnetic resonance images. Spine (Phila Pa 1976) 2010;35:191924.

23. Barz T, Melloh M, Staub LP, et al. Nerve root sedimentation sign: evaluation of a new radiological sign in lumbar spinal stenosis. Spine (Phila Pa 1976) 2010; 35:892-7.

24. Nath R, Middha S, Gupta AK, Nath R. Functional outcome of surgical management of degenerative lumbar canal stenosis. Indian J Orthop 2012;46:28590.

25. Weinstein JN, Lurie JD, Tosteson TD, et al. Surgical vs nonoperative treatment for lumbar disk herniation: the Spine Patient Outcomes Research Trial (SPORT) observational cohort. JAMA 2006;296:2451-9.

26. Amundsen T, Weber H, Lilleas F, Nordal HJ, Abdelnoor M, Magnaes B. Lumbar spinal stenosis: clinical and radiologic features. Spine (Phila Pa 1976) 1995; 20:1178-86.

27. Sirvanci M, Bhatia M, Ganiyusufoglu KA, et al. Degenerative lumbar spinal stenosis: correlation with Oswestry Disability Index and MR imaging. Eur Spine J 2008;17:679-85.

28. Sigmundsson FG, Jonsson B, Stromqvist B. Preoperative pain pattern predicts surgical outcome more than type of surgery in patients with central spinal stenosis without concomitant spondylolisthesis: a register study of 9051 patients. Spine (Phila $\mathrm{Pa} 1976$ ) 2014;39:E199-210.

29. Zheng F, Cammisa FP, Sandhu HS, Girardi FP, Khan SN. Factors predicting hospital stay, operative time, blood loss, and transfusion in patients undergoing revision posterior lumbar spine decompression, fusion, and segmental instrumentation. Spine (Phila $\mathrm{Pa}$ 1976) 2002;27:818-24.

30. Koc Z, Ozcakir S, Sivrioglu K, Gurbet A, Kucukoglu S. Effectiveness of physical therapy and epidural steroid injections in lumbar spinal stenosis. Spine (Phila Pa 1976) 2009;34:985-9. 